\title{
VII. Further remarks on the new method of determining the latitude of a place, by observations of the pole-star
}

\section{Francis Baily Esq. F.R.S.}

To cite this article: Francis Baily Esq. F.R.S. (1822) VII. Further remarks on the new method of determining the latitude of a place, by observations of the pole-star, Philosophical Magazine Series 1, 60:291, 43-49, DOI: 10.1080/147864422208652785

To link to this article: http://dx.doi.org/10.1080/14786442208652785

曲 Published online: 29 Jul 2009.

Submit your article to this journal $\pi$

ЏII Article views: 2

Q View related articles $\sqsubset$ 
VII. Further Remarks on the new Method of determining the Latitude of a Place, by Qhservations of the Pole-Star. By Francis Baily, Esq. F.Th.S.*

I $\mathrm{N}$ the last Number of the Philosophical Magazine, I pointed out the method which had been lately suggested by M. Littrow for determining the latitude of a place by observations of the zenith distance of the pole-star, at any part of its diurnal revolution. Since that paper was printed $I$ have re-considered the subject more attentively, and find that that method is not confined to the pole-star, but that it may be extended with equal advantage to other stars situated at even a greater distance from the pole, and whose slow motion in altitude renders them peculiarly adapted to such observations.

This will be seen from the following demonstration of the general proposition: and as it does not appear to me that the steps of the process have been very strictly pursued by some of those mathematicians who have treated thereon (since very rlifferent results have been deduced by them), I hope I need not apologize for here calling the attention of the reader once more to the subject.

Let $Z$ be the zenith of the place of observation; $P$ the pole; and $S$ the place of the star, in its revolution round $P$ : from $S$ let fall the line $S Q$ perpendicular to $\mathrm{ZP}$. Then we shall have $Z S=z=$ the zenith distance of the star: $\mathrm{PS}=p=$ the north polar distance of the star: the angle ZPS $=t=$ the hour angle: $\mathrm{ZP}=\dot{\psi}=$ the co-latitude of the place: and $Q P$

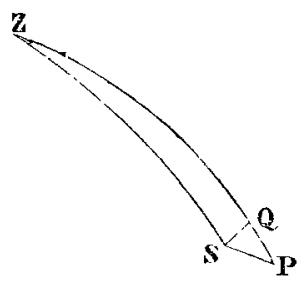
$=u=$ the segment formed by the perpendieular. Now $z, p$, and $t$ being given, we have, by the common solution of the case in spherical trigonometry,

$$
\begin{aligned}
& \tan u=\cos t \cdot \tan p \\
& \cos (\psi-u)=\frac{\cos u}{\cos p} \cos z .
\end{aligned}
$$

This solution is general: and it may readily be seen that by the help of seven logarithms, any particular case may be determined. M. Littrow has, with a view to the more familiar and easy solution of the case of the pole-star, thrown the value of $p$ and $u$ into series, by the following well known expressions:

* Communicated by the Author. 
4. Further Remarks on the new Method of determining the

$$
\begin{aligned}
\tan p & =p+\frac{p^{3}}{3}+\& \mathrm{c} . \\
\cos p & =1-\frac{p^{2}}{2}+\& \mathrm{c} . \\
u & =\tan u-\frac{\tan ^{3} u}{\mathrm{~B}}+\& \mathrm{c} .
\end{aligned}
$$

whence, by rejecting all the powers above the third, as of no value (which, in the case of the pole-star, may be safely done) and substituting the series in the general expression above mentioned, we have

$$
\begin{aligned}
\tan u & =\cos t\left(p+\frac{p^{3}}{3}\right) \\
u & =\cos t\left(p+\frac{p^{3}}{3}\right)-\frac{\cos ^{3} t \cdot p^{3}}{9} \\
& =p \cdot \cos t+\frac{p^{3}}{3}\left(\cos t-\cos ^{3} t\right) \\
& =p \cdot \cos t+\frac{p^{3}}{3} \cdot \sin ^{2} t \cdot \cos t \\
\cos u & =1-\frac{p^{2} \cdot \cos ^{2} t}{2} \\
\frac{\cos u}{\cos p^{\prime}} & =1+\frac{p^{3}}{2}-\frac{p^{2}}{2} \cdot \cos ^{2} t \\
& =1+\frac{p^{2}}{2} \cdot \sin ^{2} t \\
\cos (\psi-u) & =\cos z+\frac{p^{2}}{2} \cdot \sin ^{2} t \cdot \cos z \\
& =\cos z+\frac{p^{2}}{2} \cdot \sin ^{2} t \cdot \cot z \cdot \sin z \\
(\psi-u) & =z-\frac{p^{2}}{2} \cdot \sin ^{2} t \cdot \cot z \\
\psi & =z-\frac{p^{2}}{2} \sin ^{2} t \cdot \cot z+p \cdot \cos t+\frac{p^{3}}{3} \sin ^{2} t \cdot \cos t \\
& =z+(p+\mathrm{C}) \cos t-\mathrm{B} \cot z
\end{aligned}
$$

which is the very same formula as that which $I$ have given in my former paper.

This formula is an approximate one; but, as far as the polestar is concerned, is correct: the terms which are omitted not affecting the result in any sensible manner. If the general formula be used, it may be applied with equal advantage to $\delta$ Urse Minoris and other stars not far distant from the pole: and thus the means of deducing the latitude from such observations will be multiplied. But if that formula be converted into series, we must (in these cases) retain the fourth and sometimes the fifth powers of $p$ : which however will not render the approximate formula more intricate or laborious, and the computer may adopt it in lieu of the general one. Yet I doubt 
the advantage to be obtained thereby; as I shall in the sequel more fully explain.

I have stated that different results appear to have been obtained by different mathematicians in their solution of this problem. Dr. Dirksen makes the value to be

$$
\psi=z+p \cdot \cos t-\frac{p^{2}}{2} \sin ^{2} t \cdot \cot \psi-\frac{p^{3}}{2} \sin ^{9} t \cdot \cos t \cdot \cot ^{3} \psi
$$

which differs from the formula above adduced, by having in the third term $\cot \psi$ instead of $\cot z$; whilst the fourth term is altogether different. Mr. Horner's formula also, which is much more complex than Dr. Dirksen's, involves the cot $\psi$. The tables likewise of Mr. Stevens (to which I shall presently allude) as well as those of M. Schumacher, appear to be founded on the same erroneous formula. The table also recently published by Capt. Lynn, in page 133 of his "Star Tables for 1823," and which was furnished by an eminent mathematician and astronomer, seems formed on the same principle. The same may likewise be said of the rule given by the Rev. Mr. Lax in Problem XIV. of his "Tables to be used with the Nautical Almanac." It is not easily seen how these different authors can have deduced their formulæ so as to involve expressions depending on the co-latitude of the place of observation; since it by no means enters into any of the steps of the process. It is true that, in the case of the pole-star, and for nautical purposes, this substitution of the co-latitude for the zenith distance will not be of any consequence. But in the case of stars having greater north-polar distance, and where the observations are carried on in an observatory, the computations must be confined to the correct formula. In fact, since the true values are in all cases more easily deduced firom the correct formula, there can be no good reason for, at any time, having recourse to an erroneous one. In the example given in my former paper an error of 2 " would arise from the substitution of the approximate co-latitude, for the zenith distance: but in the case of $\delta$ Urse Minoris the error would be 10 times greater from a similar substitution *. These quantities may be of little or no moment at sea, but in an observatory they cannot be neglected.

* This star, which, after the pole-star, is the next resorted to for observations of the latitude, as well as for the adjustment of the transit instrument, is not one of the stars which are included in the Greenwich catalogue: therefore neither its mean place at the begiming of the year, nor its apparent place at any other time is to be found in the Nautical Al. manac. On the Continent, however, the position of this star is considered of so much importance that (like the pole-star) its apparent place, both in right ascension and declination, is now calculated for every day in the year: an example worthy of imitation in this country. 


\section{Frrither Rimals on the new Method of determining the}

Since the latitude may at all times be so ensily computed from the general formula which I have deduced, it does not readily appear why M. Littrow should have considered it necessary to alter the form of it, by the introduction of the series above mentioned. It is true that we are enabled thereby (as fir as regards the pole-star) to compose tables for nautical purposes, which may be of great use and advantage at sea; and save much time and labour. But, this does not seem to have been his object: and moreover, in an observatory, where the greatest accuracy is required, the approximate formula is always equally laborious with, and sometimes more so than the correct one; as may be readily ascertained on trial. For, six logarithms are always required; besides the proportional parts of $\mathrm{B}$ and $\mathrm{C}$, and other reductions on account of the variation in the north polar distance of the star, when $p$ and $t$ to not correspond with the value in the table.

'The observations, for determining the latitude of a place, are usually formed into series of 20 or 30 each: and if we could, by the help of any tables, annex to each observation its correction depending on the distance of time from the mean time of the whole series, and after dividing the sum by the whole number $(=N)$ of observations, approximate towards the correct value by the help of any formula (similar to the plan adopted by M. Delambre, in his Reduction to the meridian) a great deal of time and labour might be saved in the computations. But M. Littrow's formula presents no such advantages.

It is true that he has elsewhere snggested a plan of this kind, but I much doubt whether it can be practised with convenience or success. It is as follows: make

$$
\begin{aligned}
& m=\frac{\sin p \cdot \sin \psi}{\sin z} \cdot \sin t \\
& n=\frac{\sin p \cdot \sin \psi}{\sin z} \cdot \cos t=m \cdot \cot t \\
& \mathrm{~A}=\frac{2 \sin ^{2} \frac{\theta}{2}}{\sin \mathrm{I}^{\prime \prime}}+\frac{2 \sin ^{2} \frac{\theta^{\prime}}{2}}{\sin \frac{2}{1^{\prime \prime}}}+\frac{2 \sin ^{2} \frac{\theta^{\prime \prime}}{2}}{\sin \mathrm{1}^{\prime \prime}}+8 \mathrm{c} .
\end{aligned}
$$

where $t$ denotes the mean time of the whole number of observations, and where $\theta, \theta^{\prime}, \theta^{\prime \prime}$, \&c. denote the difference between $t$ and the time of each respective observation: then by assuming

we shall have

$$
x=\left(n-m^{2} \cdot \cot z\right) \frac{A}{N}
$$

$$
\begin{aligned}
\tan u & =\tan p \cdot \cos t \\
\cos (\psi-u) & =\frac{\cos u}{\cos p} \cdot \cos (z-x)
\end{aligned}
$$

Now, this is precisely the same formula as that which I have deduced at the commencement of this paper, with the excep- 
tion of the new quantity $x$, which is here proposed to correct the value of $z$ as deduced from a series of observations. But since the value of $x$ depends on three other formulæ, none of which are of very easy arithmetical solution, it may be doubted whether any thing is gained by the substitution. It is singular that the formula $m$ and $n$ both contain the value of $\psi$ : so that in these approximations also the latitude is supposed to be previously known before the general formula can be depended upon, The value of $m$ (if formed into a table, as proposed by M. Littrow) will necessarily require a table of double entry, even for a fixed observatory. The ralue of $A$ may be taken from M. Delambre's tables of Reduction to the meridian. M. Littrow adds, as a condition, that the observations must not be very numerous, and that if they are formed into series of four or six, we may safely reject the correction $x$ : but, by this limitation and these subdivisions, we lose the benefit of his proposed corrections, and thus revert to the original general formula. His method indeed appears to be somewhat similar to the mode proposed by M. Soldner for determining the time from the mean of a number of zenith distances of the sun or a star: (see Bode's Astronomische Jahrbuch for 1818, page 123) but which M. Delambre (in his investigation and examination of this method, in the Connaissance des Tems for 1820, pages 357 and 397) seems to consider not more convenient in practice than the rigorous formula; except in those cases where the observations are made near the prime vertical.

I shall now proceed to make a few remarks on the history of the discovery of this problem: a measure which appears to be called for at the present moment, in consequence of some observations which have been recently made on the subject by M. Zach, in his Correspondance Astronomique. Till those remarks appeared M. Littrow was (I believe) generally considered as the original inventor of the method: and in the first paper which he published on the subject in 1817, he himself calls it a new method. M. Zach, however, in a late number of his Cor. Ast. (vol. vi. page 210) states that the American sear men have been a long time in the habit of observing the altitude of the pole-star, at all hours of the night, for the purpose of deducing their latitude: and he quotes a tuble given for this very purpose, by Mr. Bowditch in the third edition of his "New American Practical Navigator." This work was originally published in America in the year 1801 ; but the third edition, here alluded to, appears without $\alpha$ date $:$ and, as it is not known whether the table was inserted in the first and second editions, no correct inference can be drawn as to the time when this method was first adopted by the American navy. 
The table given by Mr. Bowditch consists merely of $p \cdot \cos t$ converted into numbers for every $10^{\mathrm{m}}$, from $0^{\mathrm{h}}$ to $12^{\mathrm{h}}$, and on the assumption that $p$ is equal to $1^{\circ} 42^{\prime}$. No correction is given for those powers of $p$ which are omitted: and, as the polar distance of the star is assumed 4' greater than it is at the present day, it is evident that his table can give but a very rough approximation to the latitude. The American captain, from whom M. Zach obtained the above particulars, seemed aware of this circumstance, but did not consider it of much importance, because the altitude of the star was seldom determinable within those limits: an error in reasoning too frequently adopted. He said, however, that if greater accuracy were required they (the Americans) had other tables more exact; and mentioned those of Capt. Elford, of Charleston in South Carolina, published under the title of "Circular Polar 'Tables," and a work of Mr. Stevens : neither of which he had in his possession.

Capt. Elford's work I have not yet been able to. procure, neither do I know the date of it; but the pamphlet of Mr. Stevens has been kindly presented to me by Mr. Troughton; and I avail myself of this opportunity of giving a summary of its contents. It was published in this country twentytwo years ago, under the title of "A method of ascertaining the latitude in the northern hemisphere by a single altitude of the pole-star, at any time; with tables computed for that purpose. By John Stevens, in the service of the East India Company. Cambridge 1800." It contains only 16 pages; four of which are occupied by the tables. Table I. appears to contain the value of $p \cdot \cos t$ : and Table II. the correction depending on the latitude of the place of observation. The north polar distance of the star is assumed equal to $1^{\circ} 45^{\prime}$; but a rule is given for correcting the errors which may arise from any variation in that quantity.

It would thus appear (from the information which we at present possess) that this method of deducing the latitude of a place originated in this country. But, how it has happened that it should have been so much neglected here, as not to have formed a part of any subsequent treatise on navigation, whilst it has been so speedily adopted by the Americans, $I$ am at a loss to conceive. The attention, however, which has been recently directed to this subject, will probably restore it to its proper situation in all fiuture works which may be written for the information of nautical persons. The correct solution of the problem still belongs to $M$. Littrow.

M. Zach, who seems to take a pleasure in noticing the zeal and rising talents of the Americans, parlicularly in sil natutical 
affairs, has stated that Mr. Bowditch (in his "New American Practical Navigator") has re-calculated the logarithms, technically called Log. Rising, and extended them to $12^{\mathrm{h}}$. It may perhaps be gratifying to learn that a similar extension of those useful tables has also been made in this country by Captain Lynn in a work entitled "Solar Tables for working the longitude by chronometer, or for finding the latitude by double altitudes of the sun or stars." In the Requisite Tables published by Dr. Maskelyne, the Log. Rising are carried to five places of decimals, and for every 10 seconds of time. In those recently published by the Rev. Mr. Lax, they are extended to every 4 seconds of time, but still to 5 places of decimals only. In those now publishing by Capt. Lynn, they are extended to every second of time, and to six places of decimals. The work, as far as 9 hours, is already published, and will be equally useful to the astronomer and the seaman: but, whether it will be continued, or not, will probably depend on the patronage which is afforded to that part which is already before the public. Too great encouragement cannot be given to works of this kind, which abridge so much the labour of computation.

In deducing the time from altitudes of the sun or stars, by means of the strict analytical formula, we obtain $\sin ^{2} \frac{t}{2}$ as the value of the hour angle. This expression is of frequent occurrence in various astronomical solutions, and had induced me to form a table of its value for every second of the quadrant. By the help of Capt. Lynn's tables, however, this labour might have been saved, since we have merely to add the constant logarithm 5.3010300 to the logarithm of $\sin ^{2} \frac{t}{2}$, and we immediately obtain the Log. Rising: which, as I have already observed, is calculated in Capt. Lynn's tables to every second; and which consequently shows, upon inspection, the correct time required, without any further reduction. Its application would have been more general if it had contained the values of the angles in arc as well as in time; similar to the plan adopted by Mr. Lax.

VIII. True apparent Right Ascension of Dr. MasKeLYNE's 36 Stars for every Day in the Year 1822, at the Time of passing the Meridian of Greenwich. By the Rev. J. Grooby.

[Continued from vol. lix. page 101.]

The Author wishes to remark that the last six months of the year have not been calculated from the same tables as the former, but from more recent ones published in the Konigsburg Observations for 1818. The mean right ascension as there given by Bessel has also been used, instead of that given by Mr. Pond.

Vol. 60. No. 291. July 1822. G

1822. 